\title{
LETTER
}

\section{Metabolic alkalosis is the most common acid-base disorder in ICU patients}

\author{
Kjersti Mæhle ${ }^{1 *}$, Bjørn Haug ${ }^{2}$, Hans Flaatten ${ }^{3,4}$ and Erik Waage Nielsen ${ }^{1,5,6}$
}

Publications give diverging information as to which metabolic acid-base disorder is the most common in the ICU [1,2]. We explored the distribution of base excess (BE) values in a large number of ICU patients and evaluated if this distribution was related to rising sodium values after admission. BE values were obtained during ICU admission in selected periods from a first level small community hospital, a second level central hospital with university affiliations, and a third level large Norwegian university/regional hospital. Sodium values were from ICU patients in the second level hospital. Laboratory values were anonymously retrieved from databases in each hospital, aggregated and analyzed in Qlikview or Excel and exported to GraphPad Prism for column statistics and analysis of variance and for preparing graphs and frequency histograms. Anonymous laboratory data are not personal data according to Norwegian data laws and studies using such data do not need informed consent. The study was approved by the local data protection officer.

A frequency histogram for all pooled $\mathrm{BE}$ values $(\mathrm{N}=138,523)$ is shown in Figure $1 \mathrm{~A}$ and one for $\mathrm{BE}$ values divided by level of care is shown in Figure 1B. Figure $1 \mathrm{C}$ shows the rapid rise in $\mathrm{BE}$ values after admission in 118,014 samples.

Although acidosis is more common at ICU admission, bicarbonate increases over time (Figure 1D) [3]. Alkalosis can be pure or mixed. Post-hypercapnic alkalosis is a complication of mechanical ventilation in patients with chronic obstructive pulmonary disease [4].

If the count of repetitive sampling influenced our results, we assume they are skewed towards acidosis, as unstable and acidotic patients tend to have acid-base samples drawn more frequently.

Data from the Norwegian National Intensive Care Registry [5] suggest that the difference in BE values between the three hospitals in our study may partly stem from difference in patients' lengths of stay. In our study, the second level hospital had the longest median length of stay (2.7 days).

A coupling of metabolic alkalosis to rising sodium values proposed by Lindner and colleagues [6] did not seem to apply to patients in our study, as the dayby-day analyses of 118,014 sodium values after admission to the ICU in the second level hospital showed a mean of about $140 \mathrm{mmol} / \mathrm{L}$ on admission and a mean of $139 \mathrm{mmol} / \mathrm{L}$ over the following 21 days (data not shown).

To our knowledge, we have for the first time presented the distribution of $\mathrm{BE}$ values in a high number of ICU patients over their entire stay, from three hospitals of different care levels, and demonstrated an increase in $\mathrm{BE}$ values within the first days after admission. Our results show that metabolic alkalosis is, overall, the most common acid-base disorder in ICU patients.

\footnotetext{
* Correspondence: Kjmahle@gmail.com

${ }^{1}$ Department of Anaesthesiology and Intensive Care, Nordland Hospital, 8092 Bodø, Norway

Full list of author information is available at the end of the article
} 

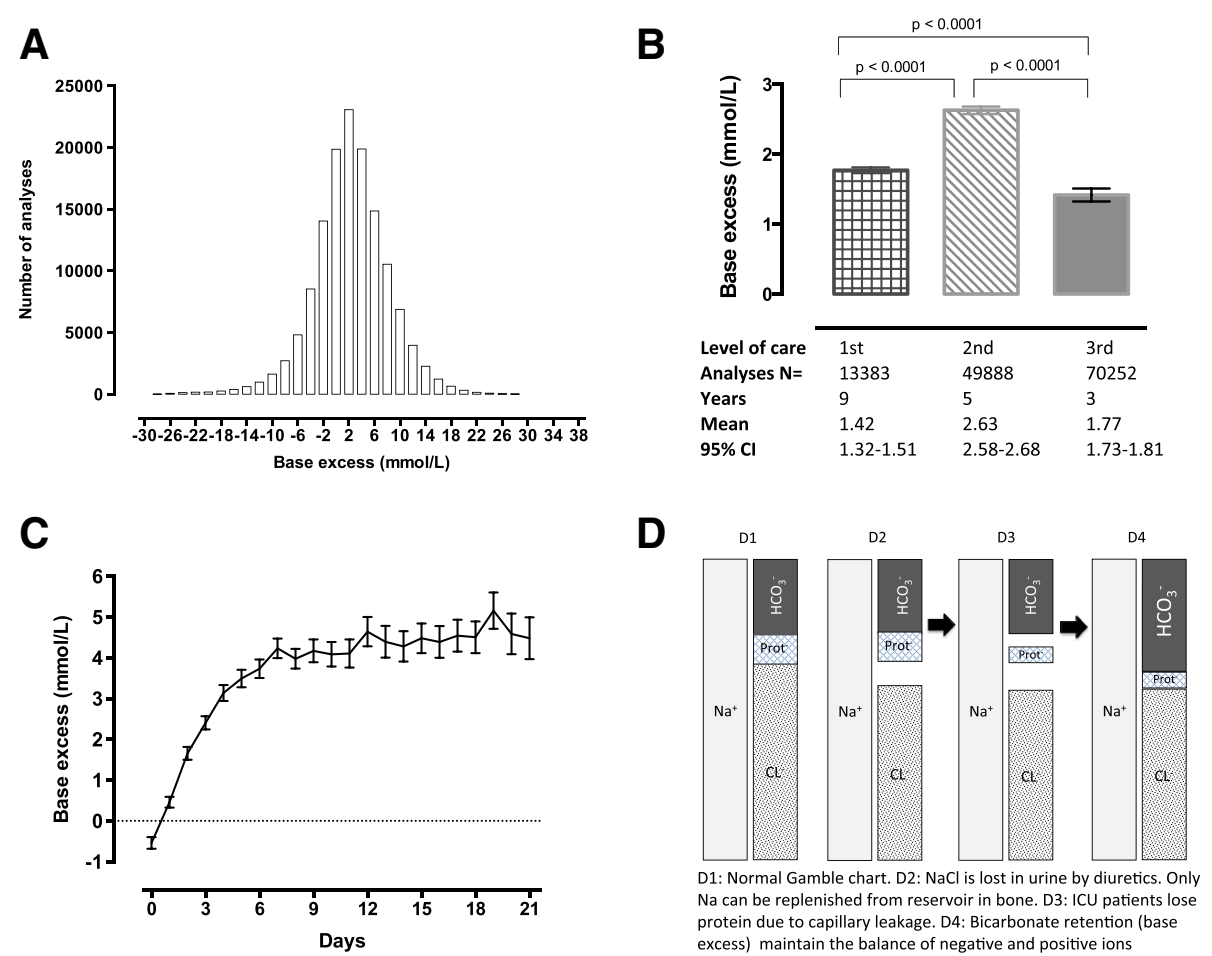

Figure 1 Distribution, between-hospital differences, development and mechanisms of metabolic alkalosis in a large number of ICU patients. (A) Frequency histogram for all pooled base excess values $(N=138,523)$ from all three hospitals. (B) Bar chart of mean base excess values with 95\% Cl from three hospitals of different care level. (C) The rise in 118,014 mean base excess values with 95\% Cl after admission to the ICU in the secondary level hospital over the following 21 days. (D) Cartoon explaining two important mechanisms behind metabolic alkalosis in ICU patients. Prot, protein.

\section{Abbreviations}

BE: Base excess.

\section{Competing interests}

The authors declare that they have no competing interests and meet the authorship requirements.

\section{Acknowledgements}

Oddgeir Strømsnes created a Qlikview file that continuously reads clinical chemistry data from the patient data system DIPS. His work is highly appreciated.

\section{Author details}

${ }^{1}$ Department of Anaesthesiology and Intensive Care, Nordland Hospital, 8092 Bodø, Norway. ${ }^{2}$ Department of Medicine, Helgeland Hospital, 8800

Sandnessjøen, Norway. ${ }^{3}$ Department of Anaesthesiology and Intensive Care, General ICU, Haukeland University Hospital, 5021 Bergen, Norway.

${ }^{4}$ Department of Clinical Medicine, University of Bergen, 5053 Bergen, Norway. ${ }^{5}$ University of Nordland, 8049 Bodø, Norway. ${ }^{6}$ University of Troms $\varnothing, 9037$

Tromsø, Norway.

\section{Published: 28 Mar 2014}

\section{References}

1. Gauthier PM, Szerlip HM: Metabolic acidosis in the intensive care unit. Crit Care Clin 2002, 18:289-308.

2. Webster NR, Kulkarni V: Metabolic alkalosis in the critically ill. Crit Rev Clin Lab Sci 1999, 36:497-510

3. Funk GC, Doberer D, Heinze G, Madl C, Holzinger U, Schneeweiss B: Changes of serum chloride and metabolic acid-base state in critical illness. Anaesthesia 2004, 59:1111-1115.
4. Heming N, Urien S, Faisy C: Acetazolamide: a second wind for a respiratory stimulant in the intensive care unit? Crit Care 2012, 16:318.

5. Norwegian national intensive care registry. http://www.intensivregister.no/.

6. Lindner G, Schwarz C, Grüssing H, Kneidinger N, Fazekas A, Funk G-C: Rising serum sodium levels are associated with a concurrent development of metabolic alkalosis in critically ill patients. Intensive Care Med 2013, 39:399-405.

\section{$10.1186 / \mathrm{cc} 13802$}

Cite this article as: Mæhle et al:: Metabolic alkalosis is the most common acid-base disorder in ICU patients. Critical Care 2014, 18:420 